\title{
INTERVENÇÃO PSICOMOTORA EM ESCOLARES COM TRANSTORNO DE DÉFICIT DE ATENÇÃO E HIPERATIVIDADE
}

\section{Siméia Gaspar Palácio}

Docente do curso de Fisioterapia do Centro Universitário de Maringá (UniCesumar), Maringá (PR), Brasil.

\section{Adriana Carla Guerra}

Fisioterapeuta pelo Centro Universitário de Maringá (UniCesumar), Maringá (PR), Brasil.

\section{Aline Vasconcellos}

Fisioterapeuta pelo Centro Universitário de Maringá (UniCesumar), Maringá (PR), Brasil.

\section{Mateus Dias Antunes}

Mestre em Promoção da Saúde pelo Centro Universitário de Maringá (UniCesumar); Especialista em Exercício Físico e Reabilitação do Idoso, pela UNIFAMMA.

E-mail: mateus_antunes03@hotmail.com
RESUMO: O presente estudo teve como objetivo analisar os efeitos da psicomotricidade sobre o desempenho motor de escolares com Transtorno de Déficit de Atenção e Hiperatividade. O mesmo foi realizado nas escolas da rede municipal de ensino de Maringá com 24 crianças de sete a dez anos diagnosticadas com o respectivo transtorno. Foram realizadas avaliações do desempenho motor utilizando o instrumento Movement Battery Assessment for Children (MABC-2). As crianças, cujo desempenho motor foi classificado como de risco e com significante dificuldade, foram convidadas a participar de um programa de intervenção psicomotora, constando de dez sessões, duas vezes semanais com duração de 60 minutos. Ao término das sessões as mesmas foram reavaliadas pelo mesmo instrumento, sendo constatado que houve diferença estatisticamente significativa nas habilidades de destreza manual $(p=0.0026)$, habilidade com bola $(p=0.0186)$ e equilíbrio $(p=<0001)$. Conclui-se que o programa de intervenção psicomotora foi efetivo para a melhora do desempenho motor das crianças com transtorno de déficit de atenção e hiperatividade, influenciando positivamente a motricidade fina, global e equilíbrio.

PALAVRAS-CHAVE: Transtorno do déficit de atenção com hiperatividade; Modalidades de fisioterapia; Promoção da saúde.

\section{PSYCHOMOTOR INTERVENTIONS IN SCHOOL CHILDREN WITH ATTENTION DEFICIT AND HYPERACTIVIT Y DISORDER}

\begin{abstract}
The effects of psychomotoricity on the motor performance of school children with attention deficit and hyperactivity disorder are investigated. Research was undertaken in municipal schools of Maringá, Brazil, featuring 24 children, aged between 7 and 10 years, diagnosed with the disorder. Motor performance assessments were undertaken with the Movement Battery Assessment for Children (MABC-2). Children with risk classification and great difficulty were invited to participate in a program of psychomotor intervention, with ten 60-minute sessions, twice a week. At the end of the sessions, the children were re-evaluated with the same tool. There was a significantly statistic difference in manual dexterity $(p=0.0026)$, capacity with a ball $(p=0.0186)$ and equilibrium $(p=<0001)$. Results show that the program of psychomotor intervention was effective for an improvement in the motor performance of children with attention deficit and hyperactivity disorder, positively affecting fine and global motoricity and equilibrium.
\end{abstract}

KEY WORDS: Attention deficit and hyperactivity disorder; Modes in physiotherapy; Health enhancement. 


\section{INTRODUÇão}

O Transtorno de Déficit de Atenção e Hiperatividade (TDAH) é atualmente considerado como um distúrbio do desenvolvimento da infância que pode persistir na vida adulta em mais de $50 \%$ das circunstâncias. Este distúrbio é observado em torno de 3 a $6 \%$ das crianças com idade escolar. A principal característica é a dificuldade em manter-se atento, a hiperatividade e a impulsividade (ROHDE et al., 2004; VERA et al., 2006).

A agitação, hiperatividade, impulsividade são mais marcantes no TDAH subtipo hiperativo impulsivo (MAJOREK et al., 2004), em que o indivíduo apresenta instabilidade de humor (TOLEDO, 2001). Esta instabilidade gera inquietação, com movimento das mãos e dos pés quando sentando, tensão da musculatura, dificuldade em ficar parado em um lugar só por muito tempo, execução de várias tarefas ao mesmo, interrupção da fala dos outros; não ter paciência para esperar a sua vez; baixo nível de tolerância e não conseguir lidar com frustrações com erros próprios ou cometidos por outras pessoas (FONTENELLE, 2001; ARAÚJO et al., 2004). Já o TDAH do tipo misto ou combinado apresenta uma combinação das características dos subtipos desatento e hiperativo-impulsivo.

Os sintomas psicomotores do quadro de TDAH formam um conjunto deficitário, levando as alterações motoras, cognitivas, de coordenação e de equilíbrio, dificultando dessa maneira o desenvolvimento infantil (ROSA NETO, 2005). A praxia é afetada, fazendo com que ocorra dificuldade da criança programar os movimentos desejados. Assim, as atividades de motricidade fina que requerem precisão e equilíbrio se veem comprometidas pelas emoções (TOLEDO, 2001).

Para uma boa resposta terapêutica, o tratamento deve basear-se em um apoio multidisciplinar. Deve ser direcionada a área emocional, pedagógica, social, cognitiva e motora, incluindo profissionais da área de psicologia, medicina, psicopedagogia e fisioterapia. O tratamento medicamentoso inclui a utilização de estimulantes, antidepressivos e medicamentos utilizados no tratamento de hipertensão com bons resultados na melhora do comportamento, no desempenho acadêmico e ajustamento social (BARKLEY, 2006).
A psicomotricidade é uma das modalidades terapêuticas utilizadas para o tratamento do TDAH. Ela é conceituada como uma ciência cujo enfoque é o movimento humano em todas as suas formas de expressão e potencialidades, quer nas suas alterações patológicas, ou nas suas repercussões psíquicas e orgânicas, com objetivos de preservar, manter, desenvolver ou restaurar a integridade de um órgão ou sistema (FONSECA, 1995). A mesma fundamenta-se em três princípios, sendo eles: a motricidade, a afetividade e a mente e volta-se à globalidade do indivíduo.

Outros autores acreditam que os déficits motores gerados pela hiperatividade são mínimos, não interferindo no desempenho funcional da criança. Sendo assim, pelas divergências apontadas na literatura a respeito dos déficits motores gerados pelo TDAH e a escassez de estudos que relacionam o transtorno a distúrbios motores, este estudo teve como objetivo analisar os efeitos da psicomotricidade no desempenho motor de escolares com TDAH .

\section{METODOLOGIA}

Trata-se de um estudo intervencional com abordagem quantitativa. O presente estudo, aprovado pelo Comitê de Ética e Pesquisa da UniCesumar, sob parecer $\mathrm{n}^{\mathrm{O}} 464.274$, foi realizado nas escolas pertencentes à rede municipal de ensino de Maringá com escolares de sete a dez anos que apresentavam diagnóstico clínico confirmado de TDAH. Para confirmação deste diagnóstico foi aplicado o questionário SNAP-IV para os pais e professores das crianças (MATOS, 2006) e uma consulta com um neuropediatra que utilizou os critérios do Manual Diagnóstico e Estatístico dos Transtornos Mentais DSM- IV-TR (JORGE, 2000), que classifica o TDAH nos subtipos: desatento, hiperativo impulsivo e combinado (POETA; ROSA NETO, 2007). Foram excluídas do estudo as crianças portadoras de deficiência visual e auditiva grave, cardiopatias, deficiência mental, deformidades ortopédicas e outros distúrbios psiquiátricos e neurológicos graves.

Após a confirmação do diagnóstico das crianças que apresentaram indicadores positivos do transtorno 
foi solicitada a assinatura do Termo de Consentimento Livre e Esclarecido (TCLE) e agendada a avaliação fisioterapêutica, que utilizou a faixa 2 da bateria Movement Assessment Battery for Children (MABC-2), destinada à avaliação do desempenho motor de crianças de sete a dez anos. Para a realização dessa avaliação foi orientado aos pais a suspensão da medicação por 48 horas antes da realização dos testes motores. O MABC- 2 é dividido em três categorias, sendo elas: destreza manual, habilidades com bola e equilíbrio estático e dinâmico, com escore padrão variando de 0 a 19 pontos, sendo que quanto maior a pontuação melhor o resultado (HENDERSON; SUGDEN; BARNETT, 2007).

A princípio somam-se os resultados dentro de cada conjunto de tarefas (destreza manual, habilidades com bola e equilíbrio) e, na sequência, somam-se esses três valores, para obter o escore total do MABC-2, que por sua vez é convertido no percentil correspondente. Escores abaixo do $5^{\circ}$ percentil são indicativos de significante dificuldade motora, entre o $5^{\circ}$ e o $15^{\circ}$ percentil representam possível risco no desenvolvimento e acima do $15^{\circ}$ percentil, indicam que a criança não apresenta dificuldade motora (HENDERSON; SUGDEN; BARNETT, 2007). As tarefas de destreza manual envolvem atividades de velocidade e precisão, as de lançar e receber (habilidades com bola) compreendem tarefas de exatidão no lançamento e recebimento de objetos, enquanto o equilíbrio estatístico e dinâmico compreende tarefas de precisão e concentração (HENDERSON; SUGDEN; BARNETT, 2007).

Para avaliação dos dados antropométricos (massa corporal e estatura), foram utilizados uma balança utilizada em superfície plana e sem calçados, posição ortostática, pés paralelos e olhar fixo no horizonte, de acordo com a orientação do pesquisador. Foi utilizada uma fita métrica, posicionado sobre o ápice da cabeça do escolar, estando descalço e com os pés unidos, voltados para frente e o olhar fixo no horizonte, orientou-se realizar uma inspiração durante a tomada da medida (LIMA et al., 2016).

Ao término das avaliações, iniciou-se o tratamento fisioterapêutico que constou de dez sessões realizadas duas vezes por semana, com 01 hora de duração, utilizando como conduta a psicomotricidade, enfocando as necessidades individuais de cada criança, conforme os resultados obtidos nos testes. Após a intervenção foram realizados os mesmos procedimentos utilizados na avaliação inicial para verificação dos resultados alcançados.

Para a análise estatística dos dados foi utilizado o programa estatístico SPSS (Statistical Package for Social Science), versão 15.0. As variáveis qualitativas foram expressas em frequências absolutas e relativas. Para análise da distribuição dos dados, utilizou-se o teste de ShapiroWilk, como os mesmos não apresentaram distribuição normal foram utilizados Mediana (Md), Média (M) e Desvio-padrão (p) para caracterização dos resultados dos dados numéricos. Para a análise comparativa entre as variáveis antes e depois do tratamento foi utilizado o teste de Tukey, estipulando como nível de significância $(\mathrm{p} \leq 0,05)$.

\section{RESULTADOS}

Em relação à caracterização da amostra, foram avaliados 24 escolares com média de idade $8,58( \pm 1,02)$ anos, dos quais seis eram do gênero feminino (25\%) e 18 do masculino (75\%). Entre o grupo de indivíduos com TDAH, 12 eram do subtipo predominantemente combinado (50\%), sete do subtipo predominantemente desatento (30\%) e cinco do subtipo hiperativo impulsivo (20\%).

$\mathrm{Na}$ análise antropométrica, verificou-se que nove crianças foram classificadas como eutróficas $(37,5 \%)$, seis como obesas (25\%), outras seis com sobrepeso (25\%) e três com magreza (12,5\%). Quanto ao desempenho motor, observou-se pelos valores das medianas que a habilidade motora mais prejudicada foi o equilíbrio, seguido da destreza manual, conforme ilustrado na Tabela 1. Ao comparar os valores das medianas da destreza manual, habilidade com bola e equilíbrio pré e pós-intervenção, observou-se melhora dos escores numéricos de cada uma destas habilidades, com resultados estatisticamente significantes, sendo o equilíbrio a habilidade motora em que se obteve maior resultado. 
Tabela 1. Resultados das variáveis do MABC-2 pré e pós-tratamento

\begin{tabular}{|l|c|c|c|}
\hline \multirow{2}{*}{\multicolumn{1}{c|}{ Variáveis (\%) }} & \multicolumn{2}{c|}{$\begin{array}{c}\text { Amostra } \\
\text { (n=24) }\end{array}$} \\
\cline { 2 - 4 } & Md. Média (pré) & Md. Média (pós) \\
\hline Destreza manual & $9(11,58)$ & $25(26,78)$ & $0.0026^{*}$ \\
\hline Habilidade com bola & $25(36,88)$ & $50(55,54)$ & $0.0186^{*}$ \\
\hline Equilíbrio & $7(12,15)$ & $43,5(43,04)$ & $<.0001^{*}$ \\
\hline Pontuação total & $56(55)$ & $75(73,21)$ & $<.0001 *$ \\
\hline
\end{tabular}

Md: mediana. *Diferença significativa $(\mathrm{p}<0.05)$.

No que diz respeito à classificação do risco e com significante dificuldade motora, bem como desempenho motor, comparando os resultados antes e após a intervenção psicomotora, observou-se através da Figura 1 a diminuição do número de crianças de aumento do número de escolares sem prejuízo motor, o que confirma os benefícios do tratamento para estes pacientes.

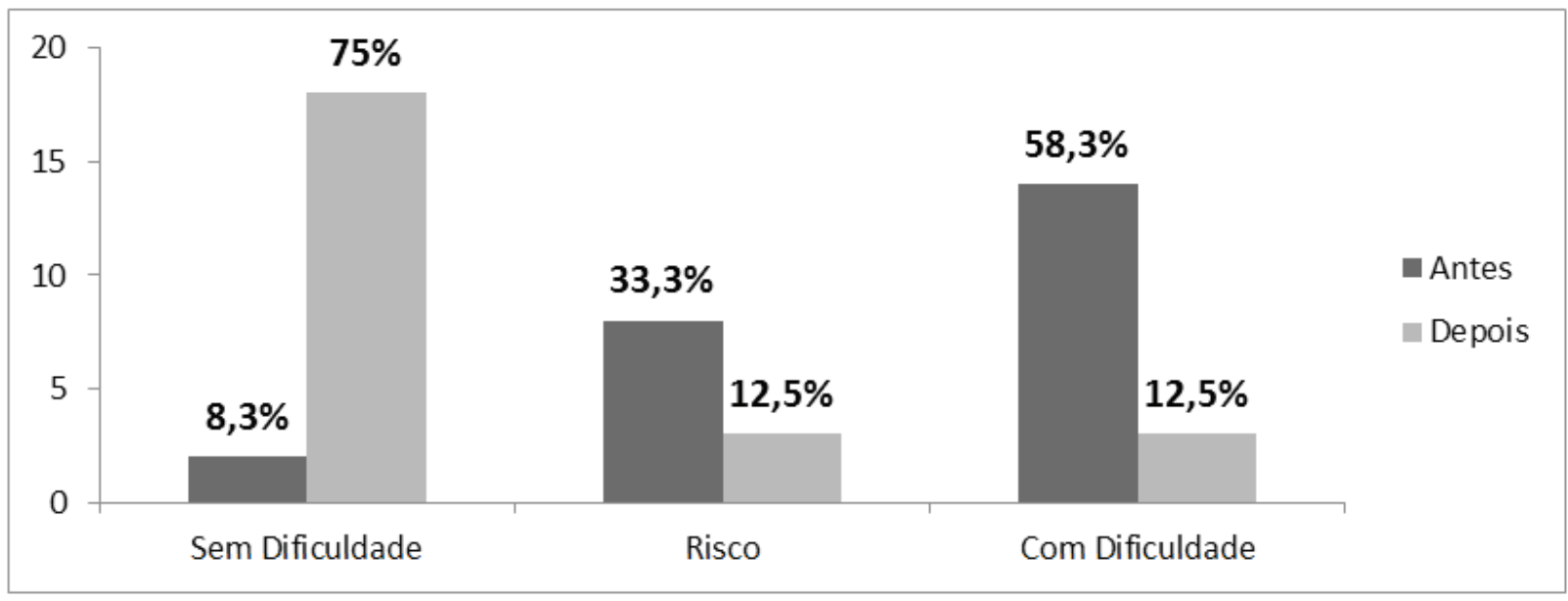

Figura 1. Classificação dos graus de dificuldade antes e após o tratamento.

\section{DISCUSSÃO}

A ocorrência de TDAH em relação ao gênero no respectivo estudo foi maior entre os meninos, o que corrobora com os achados da literatura onde também se observa maior prevalência do transtorno em indivíduos do sexo masculino (POETA; ROSA NETO, 2007; WATEMBERG et al., 2007; TONIOLO et al., 2009; VIDARTE et al., 2009; YANG et al., 2013). De acordo com Rohde e Halpern (2004), a menor prevalência de TDAH nas meninas pode ser pelo fato delas apresentarem mais comumente sintomas de desatenção e, consequentemente, menos comorbidades em termos de problemas comportamentais sendo, portanto, muitas vezes subdiagnosticadas por não causarem problemas no domicílio e no ambiente escolar (STALLER; FARAONE, 2006; STEFANATOS; BARON, 2007).

Em relação ao subtipo clínico do transtorno, neste estudo verificou-se o predomínio de crianças do tipo combinado, seguido do desatento sendo este resultado similar ao obtido por Fontana et al. (2007). Resultados contraditórios foram observados por Pondé et al. (2012) e Yang et al. (2013) que detectaram maior número de crianças do subtipo desatento na amostra que investigaram. 
Quanto ao perfil antropométrico das crianças com TDAH há resultados controversos na literatura, onde algumas pesquisas afirmam que estes indivíduos têm maior tendência ao ganho de peso (CURTIN et al., 2005), enquanto outras relatam índices semelhantes à população normal (CORTESE et al., 2010).

No que diz respeito aos benefícios da intervenção psicomotora, os resultados satisfatórios observados no presente estudo corroboram com os achados de Santos (2009) e Poeta e Rosa Neto (2005), que detectaram que a estimulação psicomotora em crianças com TDAH é relevante para o desenvolvimento da coordenação motora fina, após aplicação de 20 sessões. Lorenzini (2002) também salienta os aspectos positivos da psicomotricidade, afirmando que a brincadeira é um instrumentoquedáàcriançaaexperiêncianecessáriaaoseu desenvolvimento sensorial, motor, perceptual, cognitivo e cultural. Portanto, há evidência que o treinamento de um movimento controlado pode resultar em modificação cerebral (MAJOREK et al., 2004). Especificamente nas crianças com TDAH, a psicomotricidade passa a ser indicada para melhorar o controle motor, considerando as alterações na coordenação motora evidenciadas nessa população (FONTENELLE, 2001; TOLEDO, 2001).

Watemberg et al. (2007) reafirmam os benefícios da intervenção fisioterapêutica ao avaliarem 28 crianças com diagnóstico de TDAH associado ao Distúrbio da Coordenação Motora (DDC) com média de idade de nove anos e três meses, utilizando como modalidade terapêutica o treino de percepção motora, a terapia de integração sensorial, a cinesioterapia e a performance neurocomportamental, após oito sessões de fisioterapia com duração de 60 minutos.

Da mesma forma, Silva et al. (2011), ao aplicar um programa de intervenção motora realizados duas vezes semanais em um período de dez semanas, observaram melhora estatisticamente significativas no equilíbrio e motricidade fina o que corrobora com os achados do presente estudo. Semelhantemente Majorek, Tuchekmann e Heusser (2004) detectaram melhora nas áreas de coordenação global, equilíbrio e no comportamento social ao tratarem cinco casos de crianças com TDAH e dificuldade de aprendizagem, ao término de dez sessões com duração de 30 minutos.
A hiperatividade também diminuiu após a terapia, sugerindo que a estimulação psicomotora pode ser eficaz em crianças com o referente transtorno.

Estudos apresentam a atividade física como auxiliar no tratamento do TDAH (GAPIN; LABBAN; ETNIER, 2011; VERRET et al., 2012; ARCHER; KOSTRZEWA, 2012; MAO et al., 2014; HALPERIN; BERWID; O'NEILL, 2014). Em especial, Gapin, Labban e Etnier, em 2011, evidenciam que a atividade física proporciona efeitos benéficos sobre o desempenho cognitivo, bem como na gestão de sintomas comportamentais e melhoria do desempenho cognitivo de crianças com TDAH. Verret et al. (2012) também exploraram os efeitos de um programa de atividade física de intensidade moderada e alta na aptidão, funções cognitivas e comportamento em crianças com TDAH. Os achados evidenciaram que a participação em um programa de atividade física melhora as capacidades musculares, habilidades motoras e, segundo os pais e professores, auxilia no processamento da informação.

O desenvolvimento motor é o processo de mudanças no comportamento motor que envolve tanto a maturação do sistema nervoso central, quanto à interação com o ambiente e os estímulos dados durante o desenvolvimento da criança (OLIVEIRA; OLIVEIRA, 2006). Por isso algumas atividades podem ser incluídas na rotina escolar das crianças visando à estimulação e o aperfeiçoamento de suas habilidades psicomotoras, dando oportunidade para que por meio de atividades lúdicas, as mesmas conheçam e tenham percepção de seu corpo (BARBOSA; LIMA, 2009).

\section{CONCLUSÃO}

Por meio deste estudo, constatou-se que o programa de intervenção psicomotora foi efetivo para a melhora do desempenho motor das crianças com TDAH, influenciando positivamente a motricidade fina, global e equilíbrio. No entanto, esses resultados não podem ser generalizados em virtude da amostra reduzida e da exiguidade de trabalhos que abordam os efeitos da intervenção psicomotora nos déficits motores apresentados nos indivíduos com TDAH. Assim, salientase a necessidade de novas pesquisas com maior número 
de participantes, com intuito de favorecer a elaboração de estratégias de intervenção mais efetivas para o respectivo transtorno.

\section{AGRADECIMENTOS}

Ao Programa Institucional de Bolsas de Iniciação Científica - PIBIC/CNPq, Instituto Cesumar de Ciência, Tecnologia e Inovação - ICETI e Coordenação de Aperfeiçoamento de Pessoal de Nível Superior - Capes, pela concessão de bolsas de auxílio pesquisa.

\section{REFERÊNCIAS}

ANTONY, S.; RIBEIRO, J.P. A criança hiperativa: uma visão da abordagem gestáltica. Psicol Teor Pesq, v. 20, n.2, p.127-134, 2004 .

ARAUJO, M.; SILVA, S.A.P.S. Comportamentos indicativos do transtorno de déficit de atenção e hiperatividade em crianças: alerta para pais e professores. Rev Digital, v. 9, n. 62 , p. 1-7, 2004.

ARCHER, T.; KOSTRZEWA, R. M. Physical exercise alleviates $\mathrm{ADHD}$ symptoms: regional deficits and development trajectory. Neurot Res, v. 21, n. 2, p. 195 209, 2012.

BARBOSA, S. B.; LIMA, A. S. Psicomotricidade na educação infantil desenvolvendoCapacidades. 2 . ed. 2009.

BARKLEY, R. A. Transtorno de déficit de atenção/ hiperatividade. 3. ed. São Paulo: Artmed, 2006.

CARDOSO, F.L.; SABBAG, S.; BELTRAME, T.S. prevalência de transtorno de déficit de atenção/ hiperatividade em relação ao gênero de escolares. Rev Bras Cineantrop Desemp Hum, v. 1, n. 9, p. 50-54, 2007.

CORTESE, S.; MARCILIO, P. C. comorbidity between adha and obesity exploring shared machanisms and clinical implications. Post Grad Med, v. 122, n. 1, p. 88-96, 2010.

CURTIN, C., et al. Prevalence of overlight in children and adolescentes with attenciondéficithiperativity disorder and autismo spectrum disorders: a chart review. Bmc Pediatri, v. 4, n. 48, p. 1-13, 2005.

FONSECA, V. Manual de observação psicomotora: significação psiconeurológica dos fatores psicomotores. Porto alegre; Artmed, 1995.

FONTENELLE, L. Neurologia em adolescentes.J Pediatr, v.77, n. 2, p. 205-216, 2001.

GAPIN, J. I.; LABBAN, J. D.; ETNIER, J. L. The effects of physical activity on attention deficit hyperactivity disorder symptoms: The evidence. PrevMed, v. 52, n. 1, p. 70-74, 2011.

HALPERIN, J. M.; BERWID, O. G.; O'NEILL, S Healthy body, healthy mind?: the effectiveness of physical activity to treat ADHD in children. Child AdolescPsychiatrClin North Am, v. 23, n. 4, p. 899-936, 2014.

HENDERSON, S.; SUGDEN, D.A.; BARNETT, A. Movement assessment battery for children. 2. ed. San Antonio: Harcourt Assessment; 2007.

JORGE, M. DSM-IV: manual diagnóstico e estatístico de transtornos mentais. 4. ed. Porto Alegre: Artmed, 2000. $845 \mathrm{p}$.

LIMA, A. C. G. et al. Desenvolvimento infantil no interior do amazonas: avaliação antropométrica de escolares de 9 anos. Health Socl Chang, v. 6, n. 3, p. 42-49, 2016.

LORENZINI, M.V. Brincando a brincadeira com a criança deficiente: novos rumos terapêuticos. Barueri: Manole, 2002.

MAJOREK, M.; TUCHELMANN, T.; HEUSSER, P. Therapeutic eurythamy - movement therapy for children with attention déficit hyperactivity disorder (ADHD): a pilot study. Complem Ther Nurs Midw, v. 10, n. 2, p. 46-53, 2004.

MAO, H Y et al. Balance in children with attention deficit hyperactivity disorder-combined type. Res Develop Disab, v. 35, n. 6, p. 1252-1258, 2014. 
MATTOS, P. et al. Apresentação de uma versão em português para uso no brasil do instrumento mtasnap-iv de avaliação de sintomas de déficit de atenção/ hiperatividade e sintomas de transtorno desafiador e de oposição. Rev Psiquiatr, v. 28, n. 3, p. 290-297, 2006.

OLIVEIRA, O. R. F; OLIVEIRA, K. C. C. F. Desenvolvimento motor da criança eestimulação precoce. Fisio, v. 1, n. 2, p. 45-55, 2006.

POETA, L.S.; ROSA NETO, F. Evaluación motora em escolares con indicadores del transtorno por déficit de atención/hiperactividad. Rev Neurol, v. 44, n. 3, p. 146149, 2007.

ROHDE, L. A. et al. Transtorno de déficit de atenção/ hiperatividade na infância e na adolescência: considerações clínicas e terapêuticas. Rev Psiquiatr Clín, v. 31, n. 3, p. 45-57, 2004.

ROSA NETO, F. et al. Desempenho cognitivo e motor de uma criança com indicadores de altas habilidades. Rev Efdeportes, v. 10, n. 82, p. 1-10, 2005.

SABBAG, S.; TKAC, C.M.; BELTRAME, T.S. perfil psicomotor e os subtipos do transtorno de déficit de atenção / hiperatividade. Rev Iberoam Psicomotr Técn Corpor, v. 6, n. 4, p. 43-52, 2006.

SANTOS, M. A. et al. Psicomotricidade. Monografia (Pedagogia) - ULBRA, Santo André, 2009.

SILVA, E.V. programa de intervenção motora para escolares com indicativo de transtorno do desenvolvimento da coordenação - TDC. Rev Bra Ed Esp, v.17, n.1, p.137150, 2011.

TOLEDO, M.M. medidas para implementação de um plano de tratamento para transtorno e déficit de atenção/ hiperatividade (tda/h). Arq Neuropsiquiatr, v. 59, n. 1, p. 1321-134, 2001.

VERA, C.F.D. et al. Transtornos de aprendizagem em presença de respiração oral em indivíduos com diagnóstico de transtornos de défcit de atenção/ hiperatividade (TDAH). Rev CEFAC, v. 8, n. 4, p. 441-455, 2006.
VERRET, C. et al. A physical activity program improves behavior and cognitive functions in children with ADHD: an exploratory study. J Attent Disord, v. 16, n. 1, p. 7180, 2012.

WATEMBERG, N.; ZUK, L.; LERMAN-SAGIE, T. Developmental coordination disorder in children with attention-deficit-hyperactivity disorder and physical therapy intervention. Dev Med Child Neurol, v. 49, n. 12, p. 920-925, 2007.

Recebido em: 2017-05-27 Aceito em: 2017-08-23 